\title{
Low-Cost Prosthesis for People with Transradial Amputations
}

\author{
Haneen Mahdi Jaber ${ }^{1}$, Muhammed Abdul-Sattar ${ }^{2}$, Nabel Kadhim Abd Al-Sahib ${ }^{3}$
}

\section{Authors affiliations: \\ 1) Mechanical Eng. Dept., Al- Nahrian University, Baghdad- Iraq. hanen990990@gmail.com}

2) Mechanical Eng. Dept., AlNahrian University, BaghdadIraq. m1976sjnr@gmail.com

3) Medical Eng. Dept., AlNahrian University, BaghdadIraq.

n-k-alsahib@Y,Yahoo.com

\section{Paper History:}

Received: $21^{\text {st }}$ Nov. 2019

Revised: 12 ${ }^{\text {th }}$ Jan. 2020

Accepted: $16^{\text {th }}$ July 2020

\begin{abstract}
Prosthetic is an artificial tool that replaces part of the human frame absent because of ailment, damage, or distortion. The current activities in Iraq draw interest to the upper limb discipline because of the growth in variety of amputees and. It is necessary to do extensive researches in this subject to help lessen the struggling of patients. This paper describes the design and development of low-cost prosthesis for people with transradial amputations. The presented design involves a hand with five fingers moving by means of a gear box mechanism. The design of this artificial hand allows five degrees of freedom(5DOF), one degree of freedom for each finger. The artificial hand works by an actuation system (6V) Polou motor with gear ratio equal to 50:1 due to its compactness and cheapness. The designed hand was manufactured by a 3D printing process using polylacticacid material (PLA). Some experimental were accomplished using the designed hand for gripping objects. Initially the EMG signal was recorded when the muscle contracted in one second, two seconds, three seconds. The synthetic hand was able to produce range of gesture and grasping moves separately just like the actual hand by using KNN classification which are complete hand Pinch, fist, and jack chuck. The simulation of the fingers movements was achieved using ANSYS software to analysis the movement (pinch, fist, and jack chuck), obtain bested of stress influencer at each finger, and maximum deformation at each movement.
\end{abstract}

Keywords: Prosthetic Hand, Transradial Amputations, Gear Box, Polou

$$
\begin{aligned}
& \text { أطراف صناعية منخفضة التكلفة للمبتورين عبر الكعبري }
\end{aligned}
$$

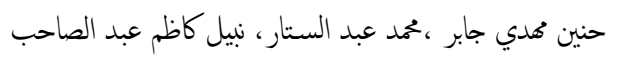

الخلاصة:

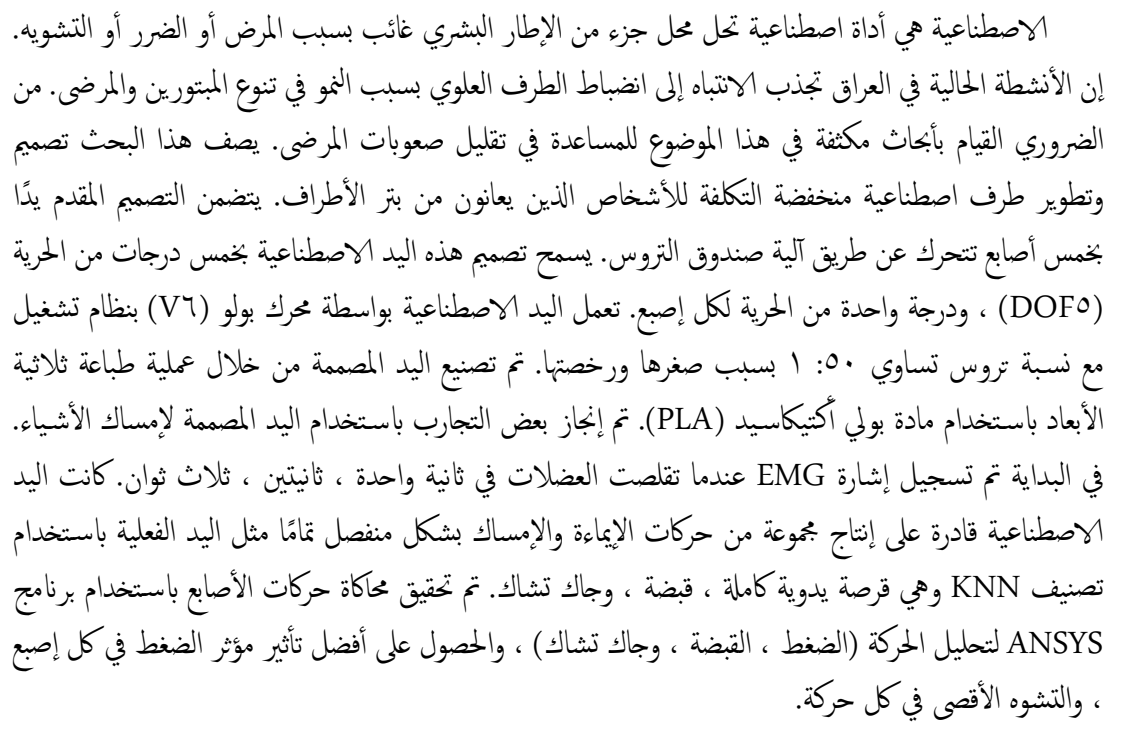




\section{Introduction}

The personal hand is an integration of complicated systems of tendons, stress, muscles, and frame. Behind a hand border, the capability of people to investigate and react to their ambiance. An unfortunate feature of a compact manual ability is the difficulty of controlling and detecting objects. Physical variations among others will result in psychological problems [1]. Estimates for persons living with us are created. More than 900,000 persons are having a slight limb loss, and 664,000 persons are having a major appendage misfortune. "Minor" appendage misfortune incorporates taking away of digits (toes or fingers) or the hand, and is the most common. "Major" limb loss includes amputation under the elbow (transradial), below the knee, higher than the elbow (transhumeral), and higher than the knee, or the foot [2]. Telemanipulation is used as an end effector for a robotic arm, which is controlled by some types of joystick and executes tasks that are inaccessible and dangerous for humans [3]. Thirty five percent of excised appendages are removed of arm or portion of arm. The arrangement and improvement of any prosthesis depends on both of the many-sided quality of the body portion being supplanted and rehabilitative necessities of the prosthesis client.

Literature review presented a new experimentalrestorative hand design that differs from the typical prostheses. With the introduction of the idea of addressing consumers necessities, practicality was increased and the cosmetic look became closer to the lost body part [4]. Some people designed a fivefingered hand with a specific number of DOF toward the person's hand. The five-fingered robot hand was designed using ultrasonic motors and elastic elements [5]. A different design to acquire a complete grasping taxonomy was presented by [6]. It composed of eight required hand postures with the possibility of controlling them by EMG signals. The design consisted of a thropomorphic hand having five fingers with 16 joints. Several studies deal with the applied forces by the hand throughout the objects griping process as mentioned by [7]. Detailed study of the mechanical properties of artificial industrial hands was performed. A pilot study on the impact of many commercially available reproductive health laboratory, including Vincent, iLimb, iLimb Pulse, Bebionic, Bebionic v2, and Michelangelo hands were reported. The design of the fingers, motility, the joint mechanical couple, and the operating methods of these artificial hands were commercially invested [8]. The stability of the mechanical design of the primary hand mechanism and, how the tradition circuit board fits within the palm was presented in [9]. Hands with fifteen degree of freedom, 7 active and 8 passives were generated and tested [10]. Several techniques to plan and make a fake hand for prosthetic application was introduced [11]. The proposed plan could be used for a five-finger hand with a lower arm incited by underactuated framework composed of ligaments and servomotors. 3D printing was used for hand fabrication. It comes woth $15 \mathrm{DOF}, 3 \mathrm{DOF}$ for each finger.
Specific gearbox was used to control and move the fingers of the artificial hand [12]. A good design for the joined curling finger was presented and developed by many teams [13-17]. The fingers differ in their precise operation and flexing mechanism style. Though every finger was primarily based around the curling life of the 6 bar linkage. Light and Chappell used a system of 5 linkages to make a mechanical curling phenomenon. [18], used a worm gear machine as an alternate of the lead screw persuaded wire system. One more advantage of the worm gear over the lead screw is that it does not need the material area of the direct separation of the wire as the wire is wound onto the machine. The equip is hence seen as an upper fashion to the coordinate screw with the cable system.

The main objective of the research is to design and manufacture an artificial hand for amputees. The place of amputation is transradial amputations and be similar in size to the human hand and equipped with sensors in order to feel the bodies. These sensors are covered with silicon in order to maintain the aesthetic of the limb. The design is implemented using six motors. The plan of an open-source, human and under actuated prosthetic hand are presented. The weight of the hand is $300 \mathrm{~g}$ which permit for an reasonable and profoundly utilitarian prosthesis gadget with a cost of $\$ 550$. Electronic design and control the prosthesis was accomplished by arduino (Nano) implemented through a sEMG activated controller and proposed in Section II. This approach allows to achieve complex customized actions, such as pinch, fist and jack chuck movement. Using the classification of motions using the Knn algorithm and also analysing stresses using the ansys program and comparing them with the stress of yielding to the material from which the prosthesis was presented in section III.

\section{Methodology}

\section{a. Geometry Design:}

This part explains the geometry design procedure used to design a mechanical function for prosthetic hand, the design of prosthetic hand was first designed and implemented using Solidworks which is a great tool to every engineer interested in designing. It is a complete Cad package that offers modeling of complicated structure as parts. The first part to design was a finger. The finger consists of two parts: distal phalange and the proximal phalange. A link between wheel gear and phalanges (proximal and distal) was introduced by a worm wheel. The $6 \mathrm{~V}$ Polou motor provides the distal motion up. The motor is linked with proximal phalange. The thumb is designed to do different functions more than any other parts design. The thumb is driven by two 6V Polou motors to provide an extension and a flexion motion. The palm is divided into two regions; one supports each finger and location of motors is called (back palm), the second part of the palm is called (upper palm). These divided parts work similar to the human hand shown in figures (1-8). 
The part forearm is designed as a base to support the palm and the rest of amputation. The wrist is designed to join the forearm with palm. All designed parts were assembled together in solidworks to form the final shape of prosthetic hand as shown in figures (1-8).

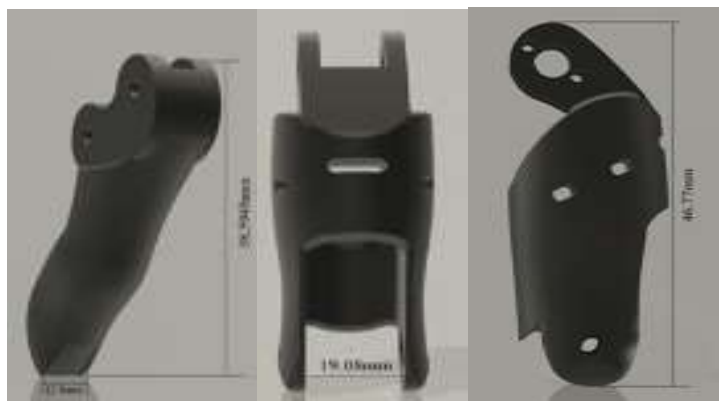

Figure (1): Parts of each finger

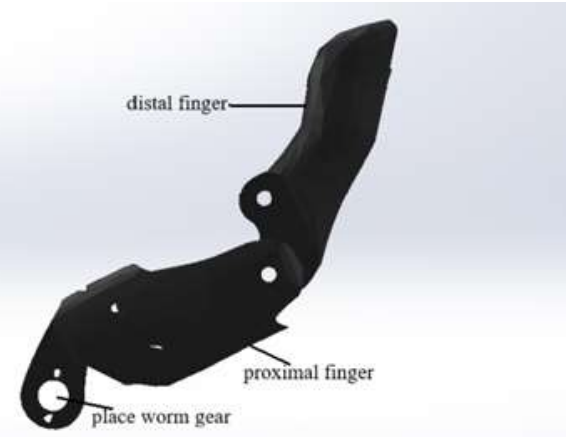

Figure (2): Index finger design

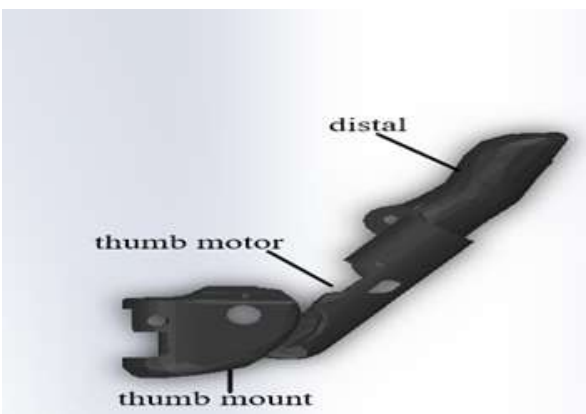

Figure (3): Thumb finger design

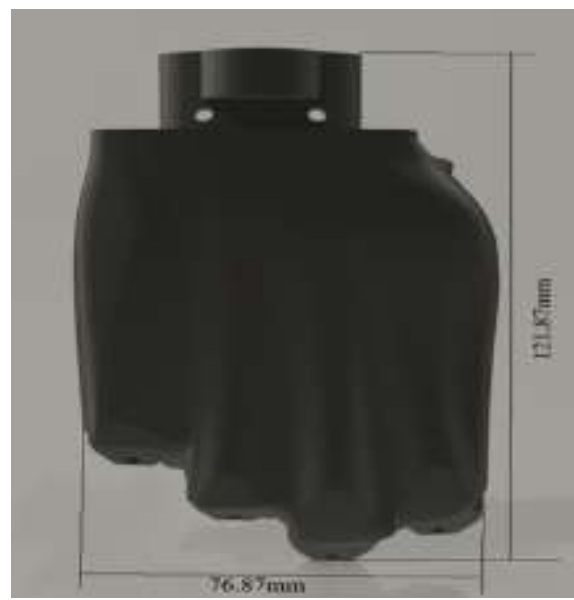

Figure (4): Back palm design

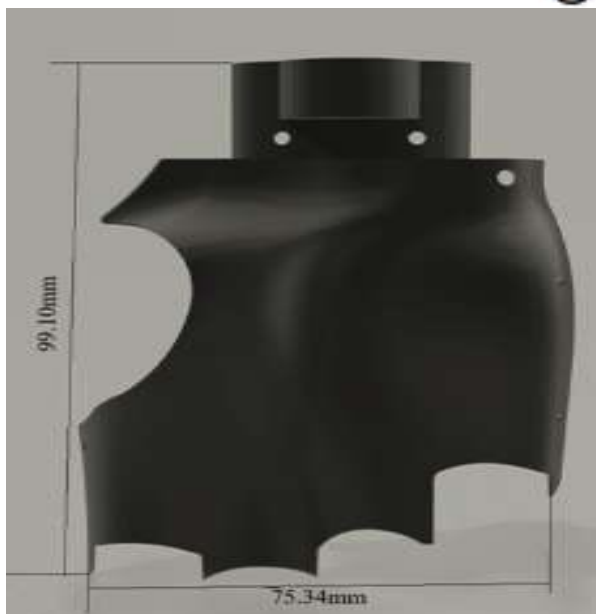

Figure (5): Upper palm design

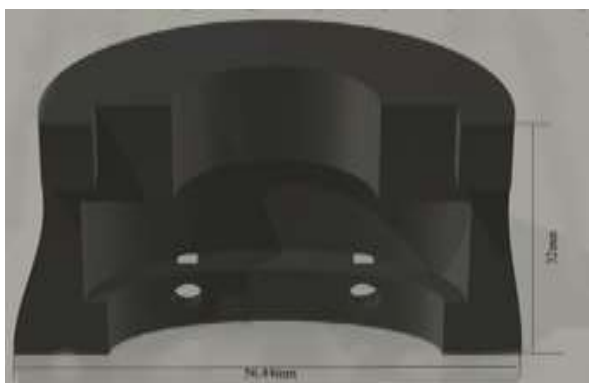

Figure (6): Wrist design

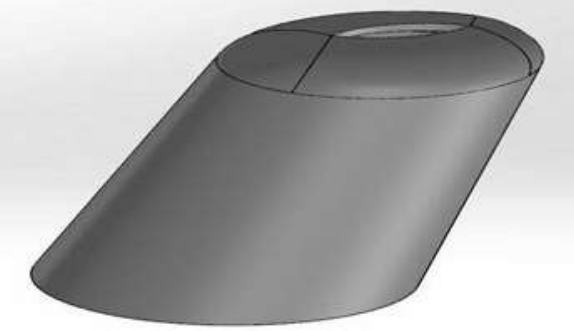

Figure (7): Part of forearm design

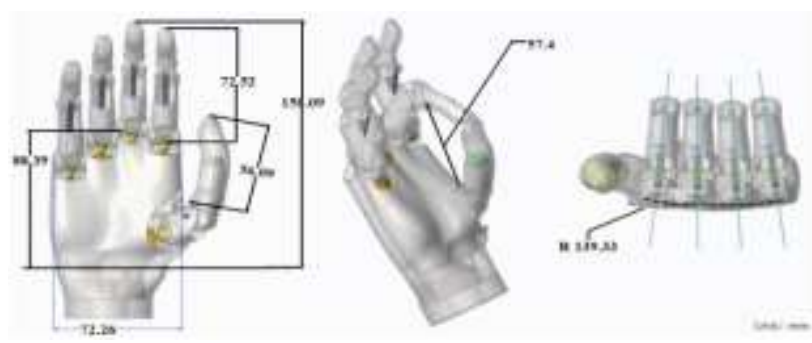

Figure (8): Complete prosthetic hand design

\section{b. Driving Mechanism:}

A gear is a device consisting of a line pipe (a worm), which mates the gearwheel (the worm wheel) in order to produce the rotational motion between the two beams at right angles to all other [19]. The worm-gear mechanism is illustrated in Fig.(9). The motor is located close to the MCP joint but requires the motor axis to be offset. The worm is mounted on the motor shaft that drives a gear mounted on the proximal 
phalangeal. Turning the worm one way or the other will control the finger motion direction [20].

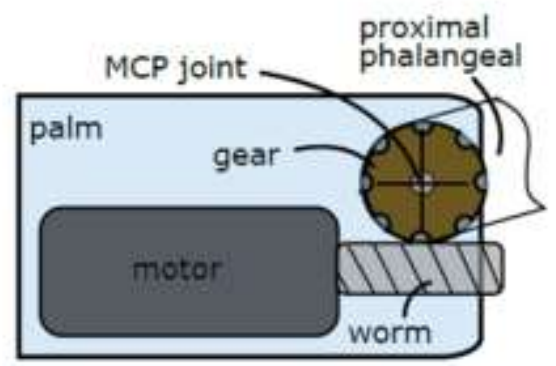

Figure (9): Worm-gear finger mechanism
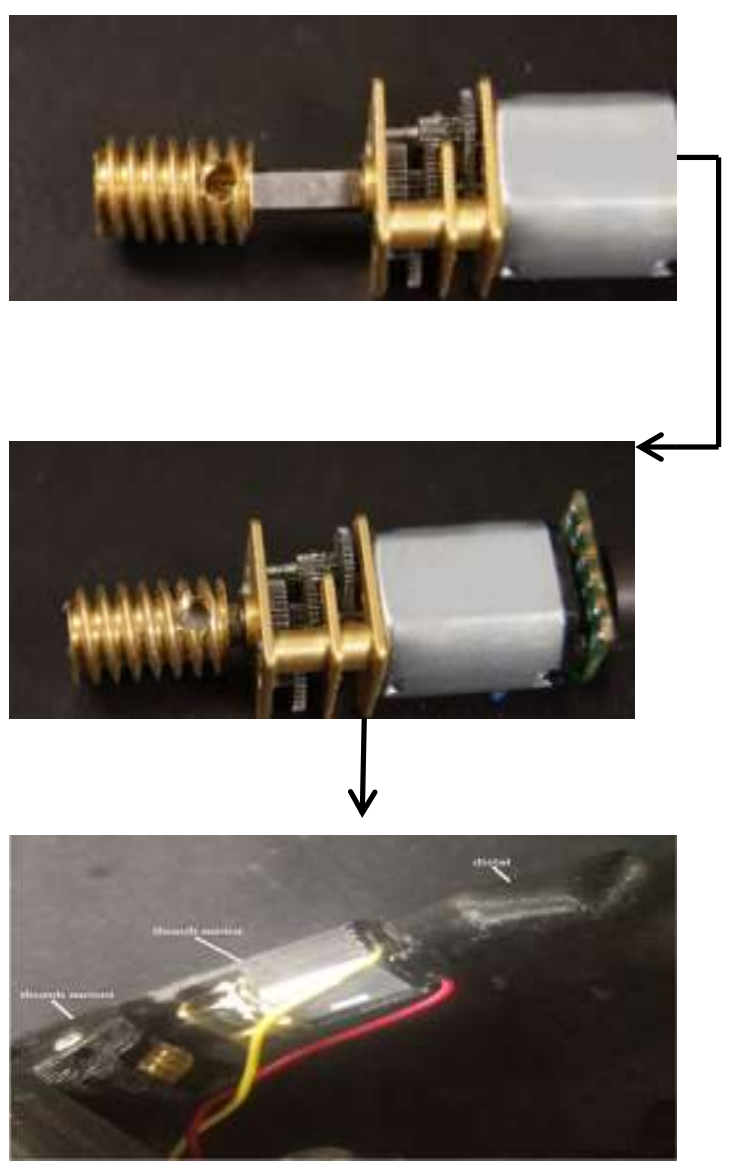

Figure (10): Motor and gear in thumb

\section{c. K-nearest Neighbor Classifier (KNN):}

K-next neighbor (KNN) could be an easy calculation that classify all cases and organize new cases that carry the same features. In addition, $\mathrm{KNN}$ algorithm is referred to as a raise the next neighbor. Next neighbor classification is mainly employed when the attributes are continuous. Easy $\mathrm{K}$ next neighbor algorithm is seen during these steps as [21]:

Step1) realize the $K$ coaching instances face to unknown example.

Step2) choose the foremost normally happening catalogue for these $\mathrm{K}$ cases.

There are numerous methods for measuring the parallel among two instances with n quality principles. Each life has taken after three arrangements. The distance (A, B) be the space among two points A, B then

1) Distance $(A, B) \geq 0$ and distance $(A, B)=0$ if $A=B$.

2) Distance $(A, B)=$ distance $(B, A)$.

3) Distance $(A, C) \leq$ distance $(A, B)+$ Distance $(B, C)$.

Chattels 3, known as "Triangle in sameness", positions that the through space between some two focuses could be a straight row. The most universal space procedure used in this study is the Euclidean space. For continuous changes, $\mathrm{Z}$ achieves equivalence, and minimum-maximum normalization is used. KNN endures from the following drawbacks: small efficiency and addiction on the range of fine charges for $\mathrm{k}$. More study is required to advance the accurateness of KNN with better charges of $\mathrm{K}$. Euclidean space between 2 focuses in $\mathrm{n}$-dimensional space is given by the condition,

Dis. $\left(x_{1}, x_{2}\right)=\sqrt{\sum_{i=1}^{k}\left(\left(x_{1 i}\right)-\left(x_{2 i}\right)\right)^{\wedge} 2}$

where:

$\mathrm{x}_{1}$ is the first point.

$\mathrm{x}_{2}$ is the second point.

$\mathrm{K}$ is the number of near neighbors.

$\mathrm{I}$ is the repetition.

where $\mathrm{x} 1=(\mathrm{x} 11, \mathrm{x} 12 \ldots \mathrm{x} 1 \mathrm{n})$ and $\mathrm{x} 2=(\mathrm{x} 21, \mathrm{x} 22 \ldots \mathrm{x} 2 \mathrm{n})$ are 2 proofs with $\mathrm{n}$ qualities. The next neighbor $(\mathrm{KNN})$ organization law assigns a model $\mathrm{z}$ of unidentified classification to the group of its near neighbor, everywhere be the near neighbor to $\mathrm{z}$ if,

$D\left(X_{i}, Z\right)=\operatorname{Min}\left\{D\left(X_{i}, Z\right) L=1,2,3, \ldots . N\right\}$

$\mathrm{D}$ is the Euclidean space among 2 models.

$\mathrm{Z}$ is a model classification.

The symbol $\mathrm{D}$ is the Euclidean space among the 2 models. The KNN rule reflects the k-near neighbors of $\mathrm{z}$ and utilizes the popular rule so that,

$\sum_{L}^{S}=1 \mathrm{t}_{1}=\mathbf{K} \quad$ This equation (c.3).

where $t$ is the time series. $S$ is the sum, $L$ is a counter: $1,2,3, \ldots \ldots N$. Then $z$ be allocated to class $j$ if

$\mathbf{t}_{\mathbf{j}}=\operatorname{Max}\left\{\mathbf{t}_{\mathbf{1}}\right\} \quad$ This equation(c.4)

where $\mathrm{j}$ is allocated to class.

The required steps on how to total K-near neighbors $\mathrm{KNN}$ algorithm are presented as,

1-Find out limit $\mathrm{K}=$ number of near neighbors.

2-Compute the space among the query-instance and the education examples.

3-Class the space and find near neighbors derived from the Kth leased space.

4-Meet the class $\mathrm{Y}$ of the near neighbors.

5-Use easy accessible of the sort of near neighbors.

\section{d. Prosthetic Hand Manufacture:}

The prosthetic hand was built using $3 \mathrm{D}$ printing as an additive manufacturing, which is a method utilized to fabricate almost any computer-aided design (CAD) model using thermoplastics materials, such as PLA, ABS, and PET. This method promises customization and simplicity with low-cost. The application of this method, to fabricate parts suited to address local needs in developing countries, has became of an interesting project in recent years. The application of $3 \mathrm{D}$ printing in low-income countries, primarily using open-source designs, holds much promise for delivering a whole 
range of desired equipment on demand. The technology has been used to deliver humanitarian aid to those in need [22].

Each finger of the prosthetic hand is actuated by $6 \mathrm{~V}$ polou motor with gear ratio equal to 50:1 located at the palm. The proposed plan of the hand was buried within the printer program and the parts were printed. The printing procedure guarantees a really tall precision when it comes to dimension; i.e, there was not much contrast between the ultimate shape measurements and the cad plan As proposed, each finger is composed of two parts connected together and fastened by pins between distal and proximal phalange. Figure (11-14) shows the finger in its final assembly. The palm on the other side was printed as two portion as planned. The fingers were associated to the palm in a really simple and straightforward way. All parts are fitted together without noticeable clearance.

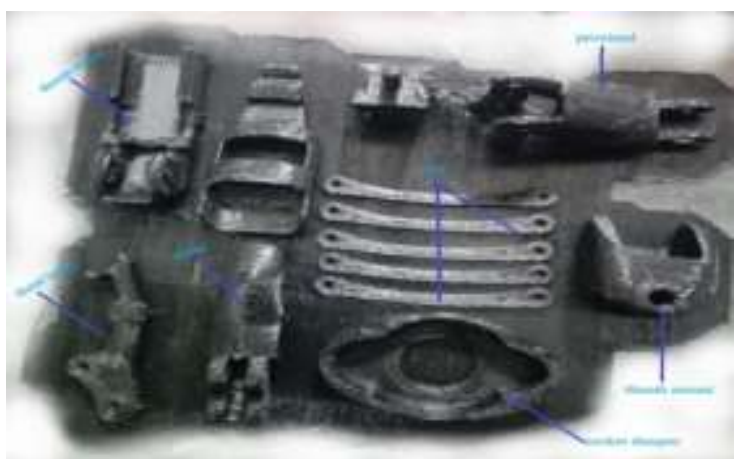

Figure (11): Part of finger

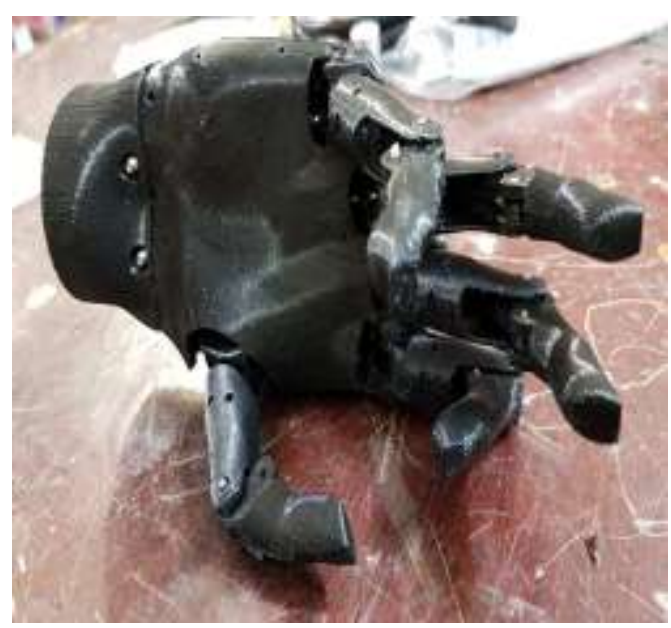

Figure (12): Palm with finger

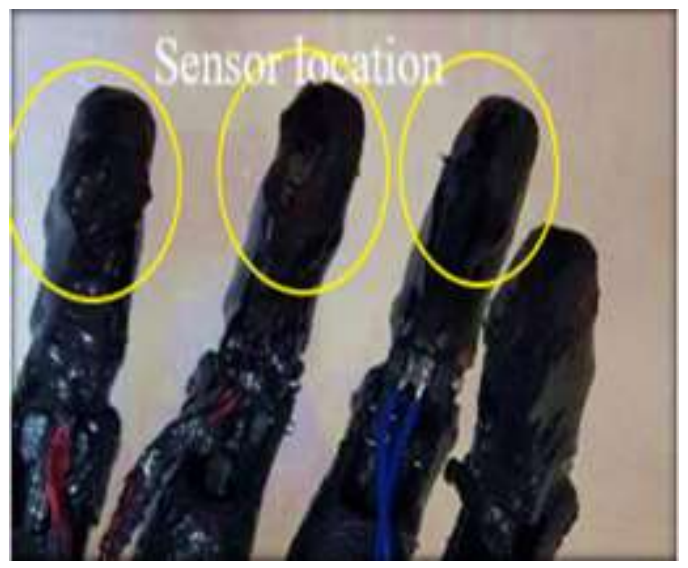

Figure (13): Sensor location at fingertip

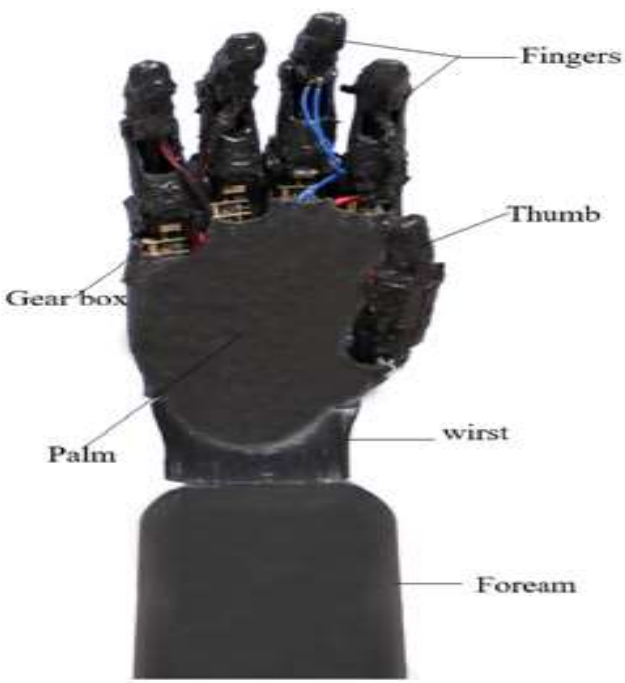

Figure (14): Fully prosthetic hand

\section{e. Setup the System Component:}

The complete control system is shown in Figure (15). The complete control system is mainly composed of EMG sensor, microcontroller, 2 DC motor drive, and the shield EMG-EKG. The shield is responsible to work as a connection part that supplies the power to the motor from a traditional $5 \mathrm{~V}$, transform and connects the battery data wires to the microcontroller pins.

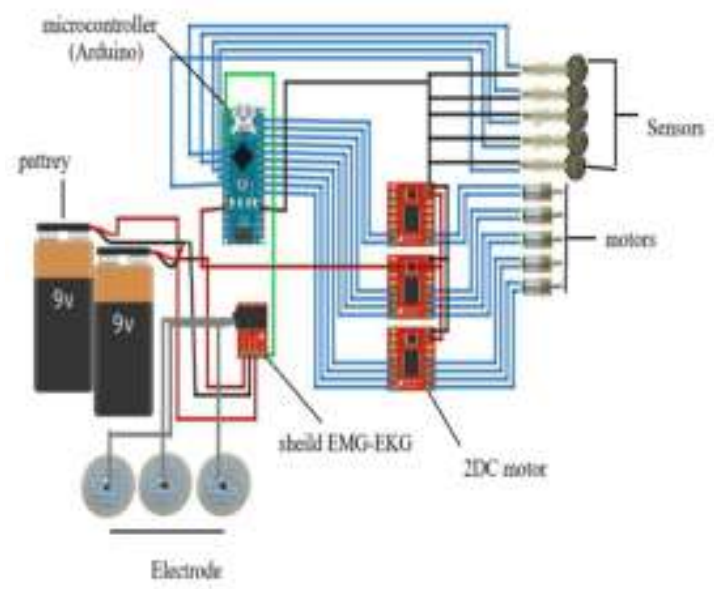

Figure (15): The complete control system.

\section{f. Surface Electromyography Signal Generation:}

The EMG signal is defined as recording and evaluating the electrical signal generated by the muscle related to neuromuscular activation. It emerges from a stream of ions across a muscle fibre's membrane passes through the connective tissues to arrive the detection electrode and it located on the skin [23] as shown in Fig.(16). The signal that passes through muscle fibres coming through axon that induces muscle contraction or influence of other neurons, action potential (AP). The motor unit action potential (MUAP) can be defined as the summation of electrical activity for all activated muscle fibres by MU. In Figure (16) the $\alpha A$ and $\alpha B$ are all alpha MUs recruited, the summation of muscle fibers APs that activated by $\alpha \mathrm{A}$ is known as $\Sigma$ MUAPA and the summation of muscle 
fibres APs that activated by $\alpha \mathrm{B}$ is known as $\Sigma$ MUAPB. This added of MUAPs in case of force increment needed. As a result of this summation of $\Sigma$ MUAPA and $\Sigma M U A P B$, the sEMG signal will emerge [24].

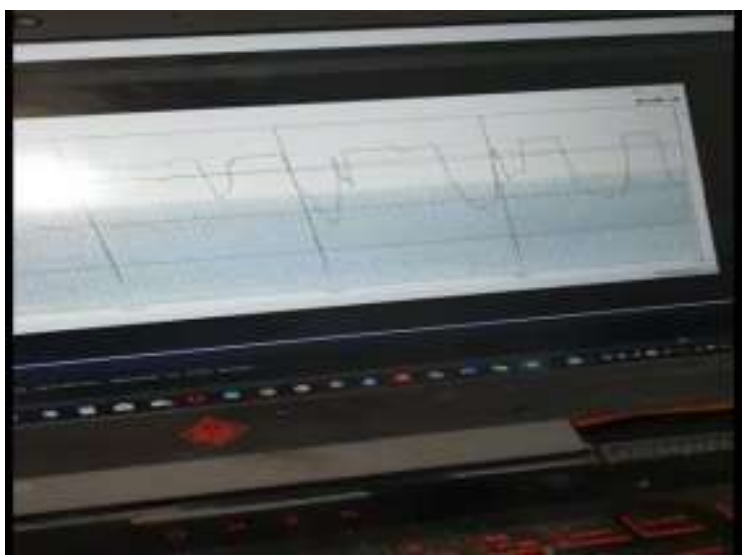

Figure (16): EMG signal generation

\section{g. Finger Kinematics:}

In the kinematics form, the prosthetic finger comprises three connections relating to the three phalanges of the true finger. The finger is set with one active and two passive DOF. The active DOF is at the main joint and the other two are in the second and third joints combined with the first. DenavitHartenberg (D-H) is the generally utilized technique to take care of the forward kinematics issue, where the change in the arrangement is utilized to create an individual change demonstrating the heading and position of the fingertip as for palm. The finger kinematics may be considered in $2 \mathrm{D}$ kinematics deceitful in the X-Y plane [25]. The D-H theorem contains four parameters which are: angle $\theta i$, the link/phalanx offset $d i$, the link/phalanx length $L i$, and the link/phalanx twist $\alpha i$.

$T_{i}=\operatorname{Rot}_{z, \theta} \times \operatorname{Trans}_{z, d i} \times \operatorname{Trans}_{x, l i} \times \operatorname{Rot}_{x, \alpha i}(3)$

$\operatorname{Rot}_{z, \theta}=\left[\begin{array}{cccc}\cos \theta & -\sin \theta & 0 & 0 \\ \sin \theta & \cos \theta & 0 & 0 \\ 0 & 0 & 1 & 0 \\ 0 & 0 & 0 & 1\end{array}\right]$

$\operatorname{Trans}_{z, d i}=\left[\begin{array}{cccc}1 & 0 & 0 & 0 \\ 0 & 1 & 0 & 0 \\ 0 & 0 & 1 & d i \\ 0 & 0 & 0 & 1\end{array}\right]$

$\operatorname{Trans}_{x, l i}=\left[\begin{array}{cccc}1 & 0 & 0 & l i \\ 0 & 1 & 0 & 0 \\ 0 & 0 & 1 & 0 \\ 0 & 0 & 0 & 1\end{array}\right]$

$\operatorname{Rot}_{x, \alpha i}=\left[\begin{array}{cccc}1 & 0 & 0 & 0 \\ 0 & \cos \alpha & -\sin \alpha & 0 \\ 0 & \sin \alpha & \cos \alpha & 0 \\ 0 & 0 & 0 & 1\end{array}\right]$

$i-\mathbf{1}_{T i}=$

$\left[\begin{array}{cccc}\cos \theta_{i} & -\sin \theta_{i} \cos \alpha_{i} & \sin \theta_{i} \cos \alpha_{i} & L_{i} \cos \theta_{i} \\ \sin \theta_{i} & \cos \theta_{i} & -\cos \theta_{i} \cos \alpha_{i} & L_{i} \sin \theta_{i} \\ 0 & \sin \alpha_{i} & \cos \alpha_{i} & d i \\ 0 & 0 & 0 & 1\end{array}\right]$

\section{h. ANSYS Analysis:}

In addition to design changes for improved cosmetics, printability, and use of equations, to design the changes for improved strength were necessary for a functional prosthetic hand [26]. The forces applied at the more critical locations and the fixed support at movement (pinch, chuck, and fist).The type and number of element are adaptive with 177660 and 108479 for node and element numbers, respectively.

\section{Results And Discussion: \\ A- KNN Classifier:}

$\mathrm{KNN}$ classifier is one of the best and the foremost vital calculation for EMG design. The calculation comprises three stages: To begin with the time separations between a test and calculation of all preparing tests. Next, choosing out the k-nearest preparing tests to the test. Finally, a course name is allotted by applying the lion's share and running the show to the $\mathrm{k}$ closest tests. In other words, $\mathrm{kNN}$ classifier is used to classify unlabelled observations by relegating them to the lesson of the foremost comparable labelled cases [27].

In $\mathrm{kNN}$ calculation the closest is characterized in terms of a time metric. Euclidean separate is utilized here between each three pattern times where the first pattern is assigned as a set between $(0.5-1.4) \mathrm{sec}$. The second is assigned as a set between $(1.5-2.4) \mathrm{sec}$. The third is assigned as a set between $(2.5-3.4)$ sec. These time values are represented as muscle contraction time. The three clustering results are shown in Fig.(17) where the blue cluster presents the pinch grip, the red cluster presents chuk grip, and the green cluster presents fist grip.
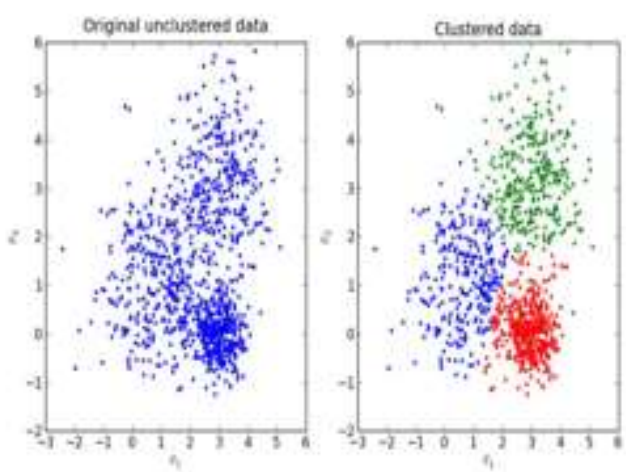

Figure (17): KNN algorithm with three clusters

\section{B- Grasp Mode:} (pinch, chuck, and fist)

The classification execution is gotten for the highlight vector time set [pinch / chuck / fist] using KNN classifiers. After gathering handle of prosthetic hand primary parts, the proposed hand is tried in a hold design. Within the grasp design test, the hand is tried to perform three hold designs utilizing EMG as flag input. The prosthetic hand can effectively perform 
three hold designs. The three grasp designs are pinch grasp, chuck, and fist thumb.

\section{Pinch grip:}

The contraction of the muscle in the pinch grip has period time of $1 \mathrm{sec}$ for transmitting it using the EMG signal, the KNN distinguishes these contractions to recognize which of these grips are done and divided into three clusters, each cluster represents one of these grips. The grip of holding the card or key are presented in Figures (18) and (19). The measuring of the amount of pressure placed on these objects can be implemented through the pressure sensors at the end of each finger as shown in Figure (20).

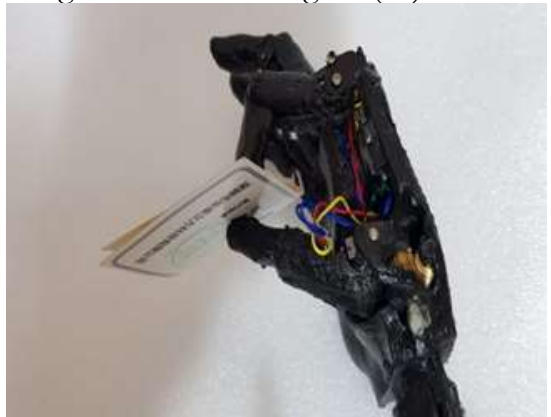

Figure (18): Hand holding a card

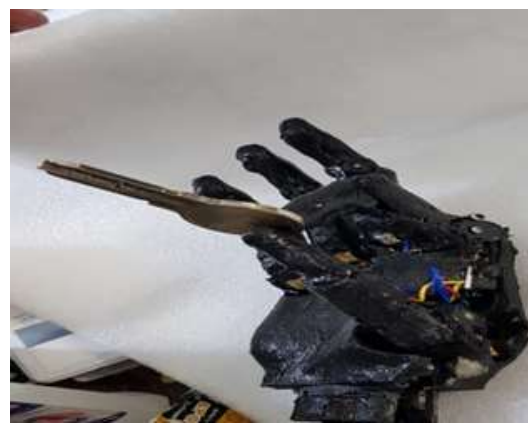

Figure (19): Hand holding a key

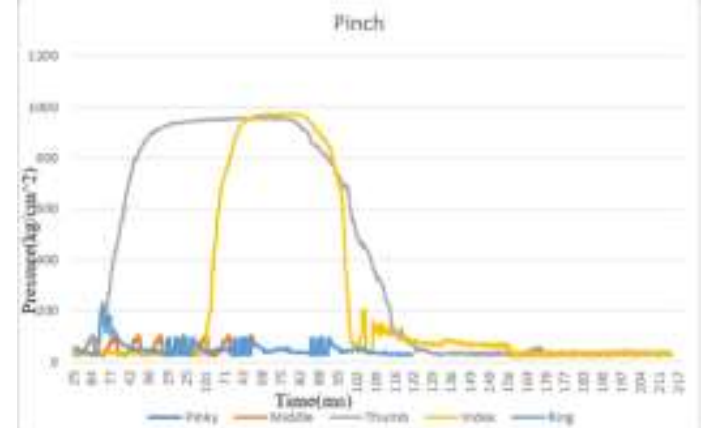

Figure (20): Value of pressure sensor with pinch

The simulation for the fingers was accomplished by ANSYS software for the pinch grip. The maximum stress for each finger is presented in Figures (21) and (22). The compression stress is maximum stress at the contact region and noticed to be $1019 \mathrm{~Pa}$ at the index finger. The second finger (thumb) has greater compression stress of maximum value of $1056.2 \mathrm{~Pa}$. The compression stress on the thumb is greater than that corresponding in the index, because the surface area of the contact is greater. Compared to the stress of subjecting the material from which the prosthesis was made, the stresses generated in each finger do not exceed the value of the stress of subjecting the material which is $59 \mathrm{Mpa}$.

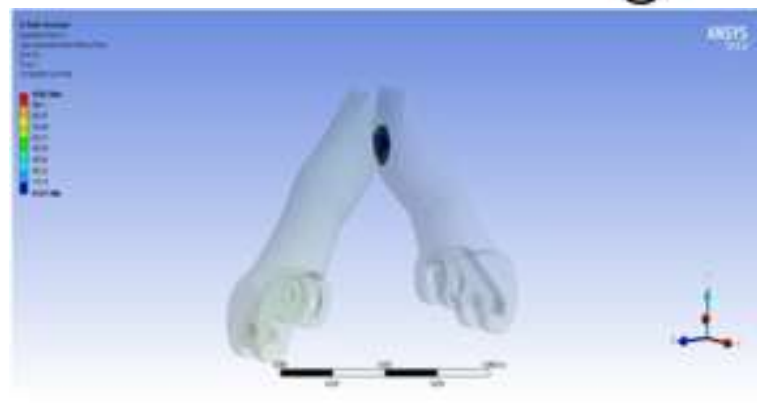

Figure (21): Maximum stress at index finger for pinch grip

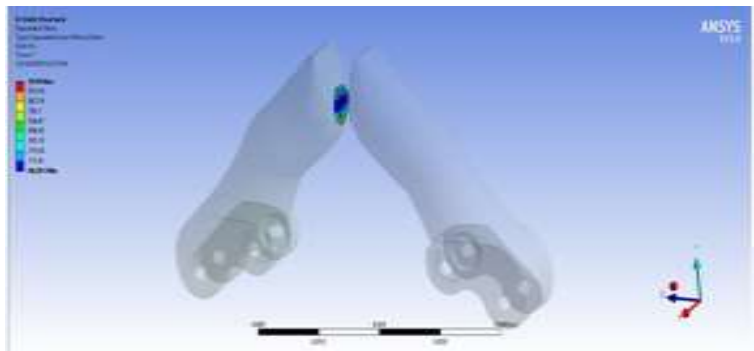

Figure (22): Maximum stress at thumb finger for pinch grip

The maximum deformation is taken at joints of finger parts. Maximum value is $1.722 * 10-8 \mathrm{~m}$, so the material of these parts must be strong. The maximum deformation is presented in Figure (23).

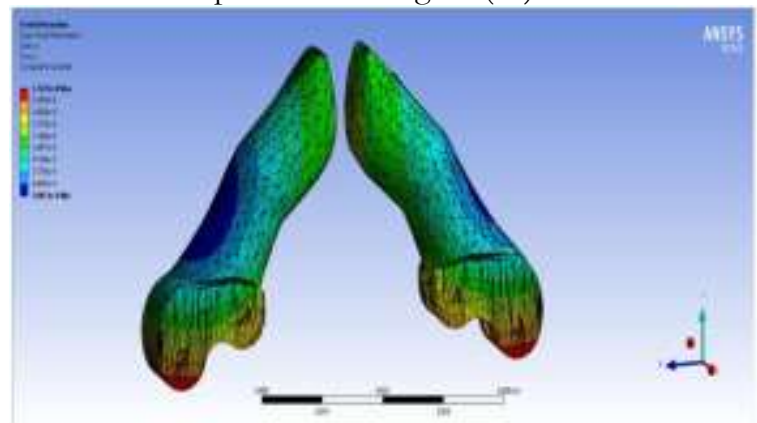

Figure (23): Maximum deformation at pinch grip

\section{Chuck grip:}

The contraction of the muscle in the chuck grip has period time of $1 \mathrm{sec}$ for transmitting using the EMG signal, the KNN distinguishes these contractions to recognize which of these grips are done and divided into three clusters each cluster represents one of these grips, the grip of holding a screwdriver is shown in Figure (24). Figure (25) shows that the pressure is developed only at the thumb, index, and middle finger, while the pressure the other fingers equals to zero (not used in this process).

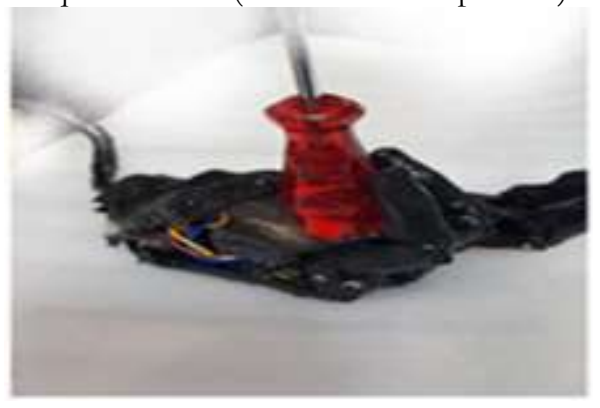

Figure (24): Prosthetic hand with chuck function 


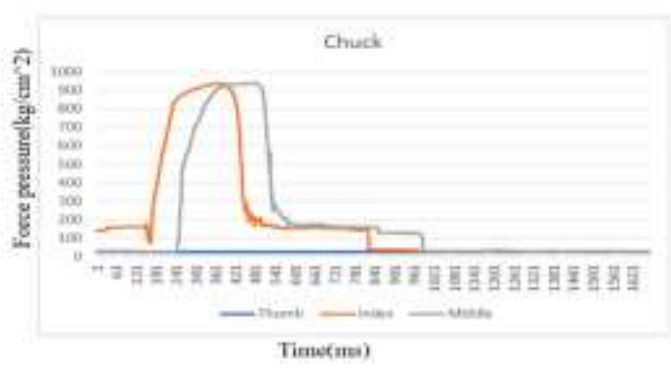

Figure (25): Value of pressure sensor with chuck

For the chuck grip, three fingers are used. The maximum stress for each finger are presented in Figures (26-28). The maximum stress distribution is measured at the contact region mainly at thumb finger with maximum stress of $1112.3 \mathrm{~Pa}$ with state of catch screw driver. The maximum stress distribution of index finger is $1002.3 \mathrm{~Pa}$ at contact region. For the middle finger, the maximum stress distribution at contact region is $1013.8 \mathrm{~Pa}$. The stresses generated in each finger do not exceed the value of the stress of subjected material which is $59 \mathrm{Mpa}$.

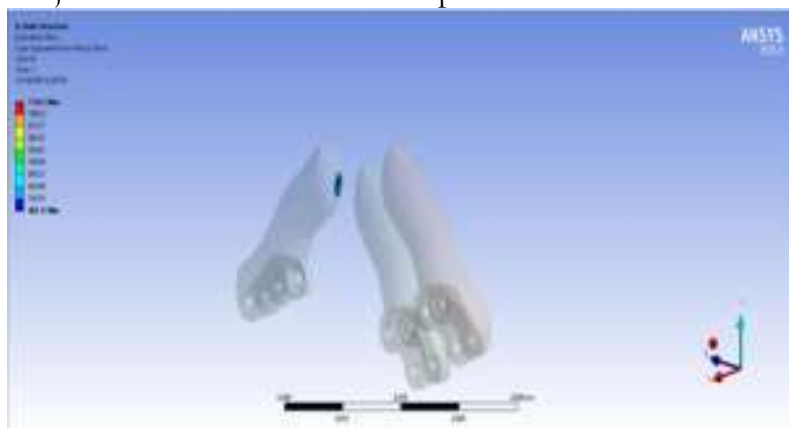

Figure (26): Maximum stress at thumb finger for chuck grip

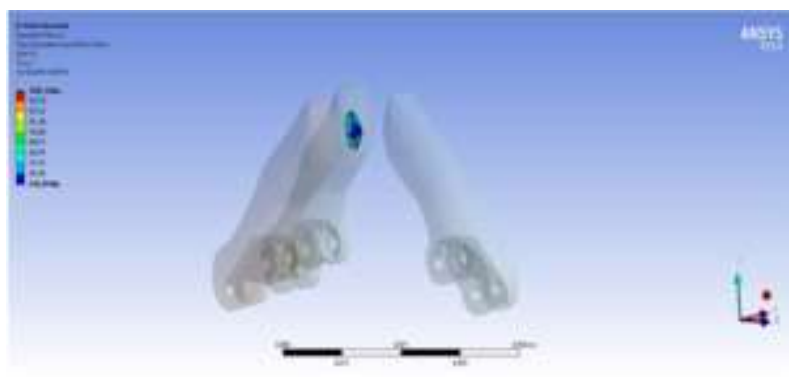

Figure (27): Maximum stress at index finger for chuck grip

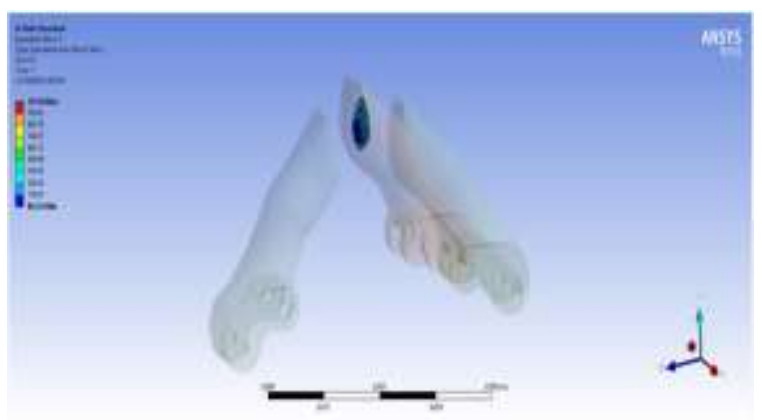

Figure(28): Maximum stress at middle finger for chuck grip
The maximum deformation is measured at joint of thumb finger with value of $4.7 * 10-9$ as shown in Figure (29).

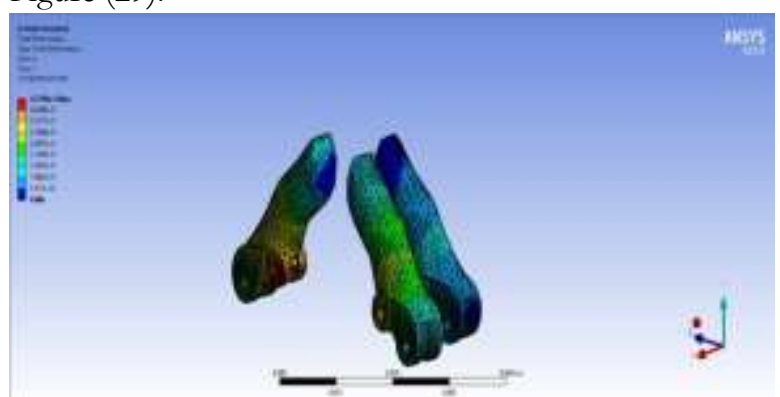

Figure (29): Maximum deformation at chuck grip

\section{Fist grip:}

The contraction of the muscle in the first grip has period time of $1 \mathrm{sec}$ for transmitting it using the EMG signal, the KNN distinguishes these contractions to recognize which of these grips are done and divided into three clusters, each cluster represents one of these grips. The grip of holding the bottle is presented in Figure (30). Measuring of the amount of pressure placed on these objects can be implemented through the pressure sensors at the end of each finger as shown in Figure (31). This figure shows that the pressure is developed at the index, ring, middle, and pinky finger, while no developed pressure noticed at the thumb finger (not used in this process).

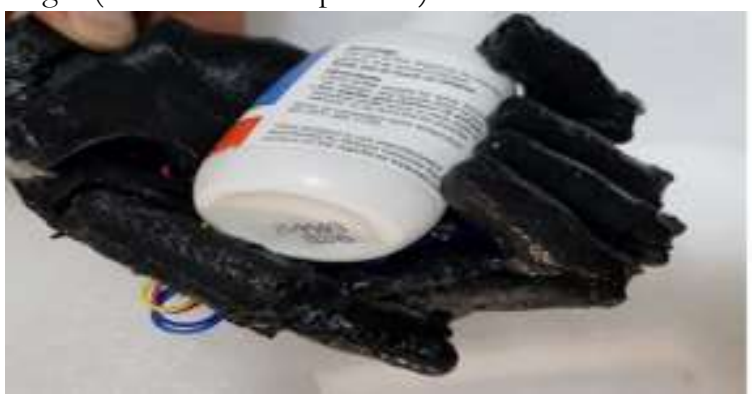

Figure (30): Prosthetic hand with fist function

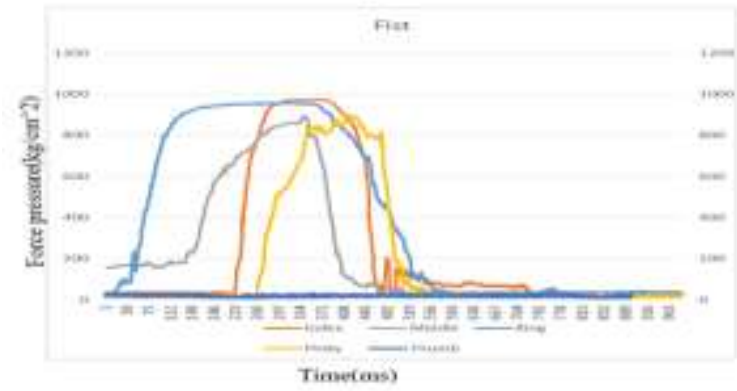

Figure (31): Value of pressure sensor with fist

Four fingers are used in this grip. Maximum stress for each finger is presented by Figure (32-35). The maximum stress distribution found at the wrist joint is 998.59 $\mathrm{Pa}$ for the index finger, 1215.4 Pa for middle finger, 1082.2 $\mathrm{Pa}$ for ring finger, and 1392.3 Pa for the little finger. The stresses generated in each finger do not exceed the value of the stress of subjected material which is $59 \mathrm{Mpa}$. 


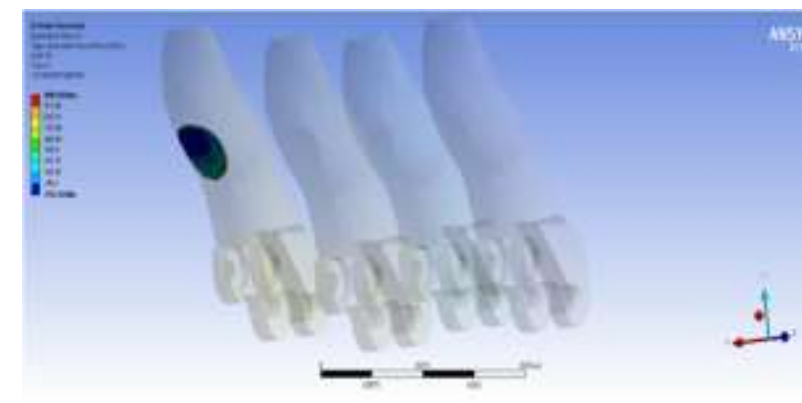

Figure (32): Maximum stress at index finger for fist grip

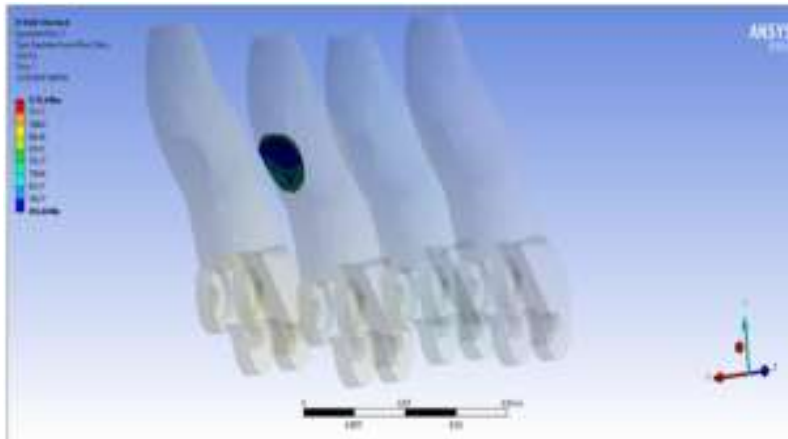

Figure (33): Maximum stress at middle finger for fist grip

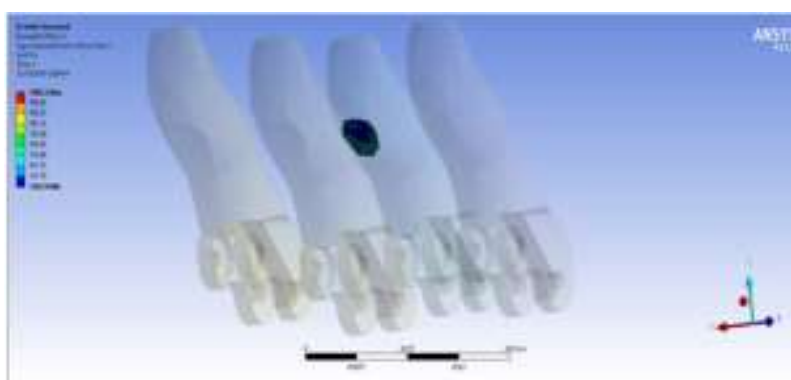

Figure (34): Maximum stress at ring finger for fist grip

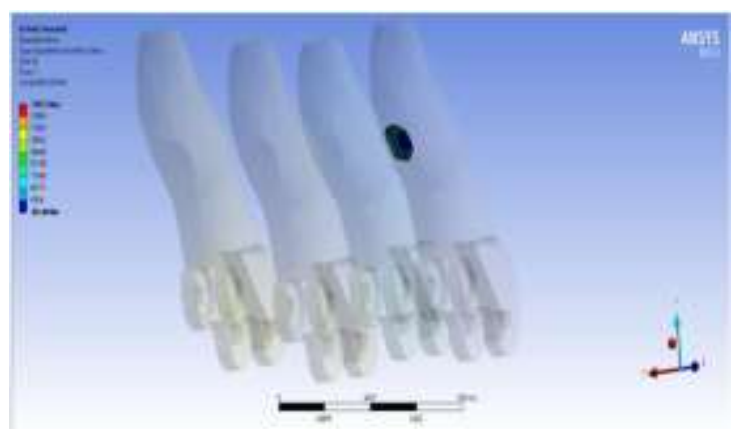

Figure (35): Maximum stress at little finger for fist grip

Seen from figure (36), the maximum deformation is taken at joint of middle finger with value $\left(8.613^{*} 10^{\wedge}\right.$ $11 \mathrm{~m})$.

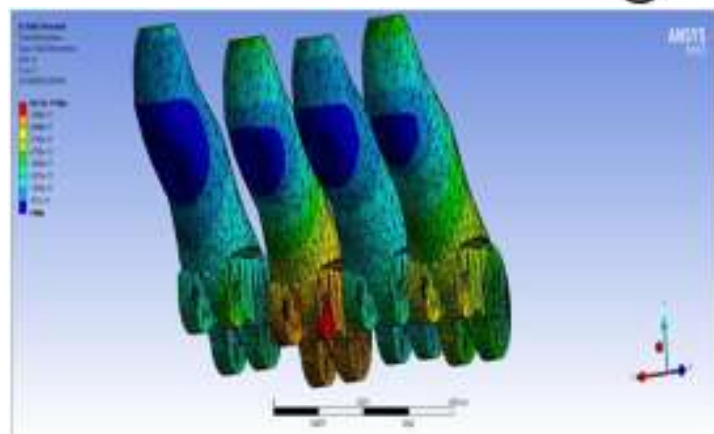

Figure (36): Maximum deformation at fist grip

The results obtained from ANSYS software are in good agreement with practical implementation of the hand.

\section{A transradial amputee live test:}

On a transradial male amputee, the prosthesis hand was tested. The amputee was without any experience to use prosthetics powered by EMG. Before using the prosthesis hand (including calibration), the amputated person was exercised for 30 minutes. This learning cycle is demonstrated in Figure (37). That procedure allowed the amputee to concentrate on the correct muscles and map the movement of the musculoskeletal to stabilize the prosthetic hand. Because of the subject's amputation, the two EMG electrodes are mounted over his triceps and biceps. After 30 years of amputation, the amputee did not use these muscles at all, and thus a significant effort had been spent to enable him using the prosthetic hand.

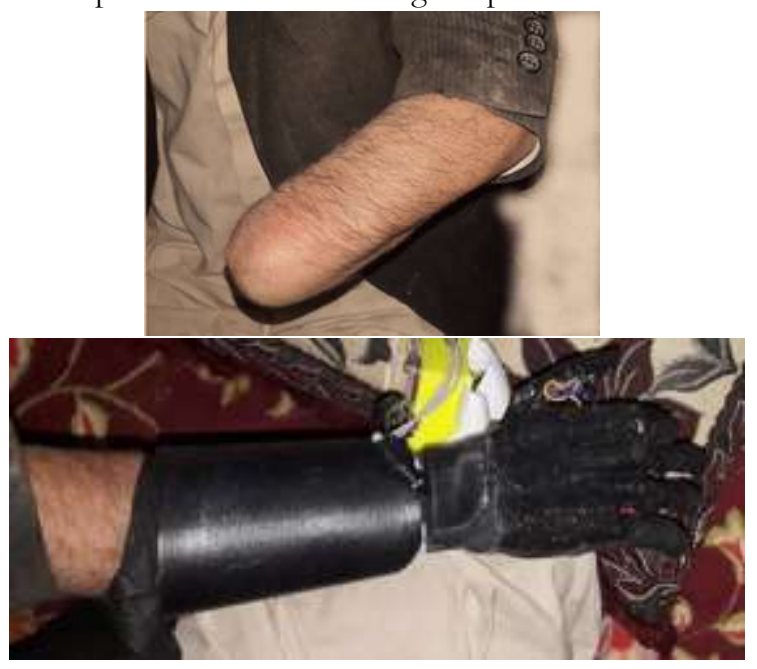

Figure (37): Wearing a prosthesis from an amputated person

\section{Conclusion:}

The prosthetic hand presented in this work was factory-made via $3 \mathrm{D}$ printing strategies. The fingers unit was accomplished by Polou 6V DC motor through a worm equip and worm wheel close to the finger. The five fingers, wrist and the attachment have five independent degrees of opportunity. Each finger is controlled by one motor while the thumb is controlled by 2 motors. The DC motor is mounted in palm. After collecting the signals for contracting the muscle for a period of one, two and three seconds 
using EMG the motions (pinch, fist, jack chuck), it classified using the nearest neighbour ( KNN) algorithm. Euclidean distance is used between each of the three pattern times where the first pattern is assigned as a set between $(0.5-1.4) \mathrm{sec}$. The second is assigned as a set between $(1.5-2.4)$ sec. The third is assigned as a set between (2.5-3.4) sec. The highest pressure was measured to be $1000 \mathrm{~kg} / \mathrm{cm} 2$. Then the fingers were analyzed by ANSYS software at similar movements to obtain the highest possible stress. It was noted that the new design gave faster response, easy to control, and can easily modified. In addition, the cost is relatively low. The stresses measured at the fingers tip do not exceed the yield stress of material under test. Depending on the signals and feedback provided by the pressure sensors, the designed hand has successfully accomplished the test of gripping different samples. The warm gear mechanism and the DC motors selection was a good choice for controlling the fingers rotational motion.

\section{REFERENCE}

[1] J. S.Micera, (2010). ,"Control Of Hand Prostheses Using Peripheral Information",. IEEE Reviews In Biomedical Engineering, vol. 3. No.1, pp.4868.

[2] D. A. J.Kurichi, (2012)., "Factors Associated With Home Discharge After Rehablitation Among Male Veterans With Lower Extremity Amputation",. Pm\&R, vol. 5, no. 5, pp. 408-417.

[3] S.F.Hasan. (2017)., "Design Of Anthropomorphic Artificial Hand For Prosthetic",. Iraq: University Of AL-Nahrain Collage Of Enginnering .

[4] S.C.Pylatiuk, (2004)., "Progress In The Development Of A Multifunctional Hand Prosthesis",. Proceedings Of 26th Annaual International Conference. San Francisco: IEEE Embs CA, USA, September 1-5.

[5] I. A.T.Maeno, (2005)., "Five-Fingered Robot Hand Using Ultrasonic Motors And Elastic Elements",. IEEE International Conference On Robotics And Antomation. Barcelona, Spain.

[6] E.Tumas.Wiste. (2009)., "Design Of A Multifunctional Anthropomorphic Prosthetic Hand With Extrinsic Actuation",. IEEE Conference On Rehabilitation Robotics. Japan.

[7] D. Rocha, (2011)., "Grip Force Analysis In A 3D Hand Prosthesis Model",. Brazil: Brazilian Congress Of Mechanical Engineering.

[8] T.Joseph.Ms.Belter, L. (2013).", "Mechanical Design And Performance Specifications Of Anthropomorphic Prosthetic Hands", JRRD, Vol.50, No.5, pp. 559-618.

[9] R.G.K.Jones, (2016)., "Mechanical Design And Development Of The Touch Hand II Prosthetic Hand",. R\&D Journal Of South African Institution Of Mechanical Engineering, ISSN 0257-9669,pp. 23-24.
[10] A. E. D.S.V.Bandara, (2017)., "Development Of A Multi-DOF Transhumeral Robotic Arm Prosthesis",. Elsevier,pp 131-141.

[11] N.Kadhim, et al (2018)., "Design And Manufacture A 3D Printed Artificial Hand",. eISSN: 2320-0847 p-ISSN : 2320-0936, pp. 156164.

[12] J.Guo, (1993)., "Optimal design of a six-bar linkage with one degree of freedom for an anthropomorphic three-jointed finger mechanism",. Journal Of Engineering In Midicine, vol. 207, no.3, pp185-190.

[13] M. E. M.H.Asyali, (2011)., "Design And Implementation Of A Voice-Controlled Prosthetic Hand",. Elektric-Turkish Journal Of Electrical Engineering And Computer Sciences, vol. 19, no.1, pp.33.

[14] E. A. N.Dechev, (2001)., "Multiple Finger, Passive Adaptive Grasp Prosthetic Hand",. In Mechansim And Machine Theory vol.36, no.10, pp.1157 1173.

[15] P. A.Kyberd, (1994). ",The Southampton Hand: An Intelligent Myoelectric",. Journal Of Rehabilitation Research And Development,vol. 31,no. 4,pp.326-334.

[16] C. A. P.J.Kyberd, (2001)., "The design of anthropomorphic prosthetic hands: A study of the southampton hand",. Robotics. VOL. 19, no.06, pp.593-600.

[17] A. A. L.Tang, (2011)., "Hand With A Simple Driving System In Robotics",. Automation And Mechatronics, pp. 208 -213.

[18] S.V.D.Riet, (2015)., "The Low-Cost Design Of A 3D Printed Multi-Fingered Myoelectric Prosthetic Hand",. Nova Science Publishers Inc, pp.784-801.

[19] Collins English Dictionary - Complete and Unabridged, 12th Edition 2014 , HarperCollins Publishers 1991, 1994, 1998, 2000, 2003, 2006, 2007, 2009, 2011, 2014/ https://www.thefreedictionary.com/worm+gear.

[20] G.K. Jones and R. Stopforth(2015)," Mechatronic Design And Optimisation of a low-cost prosthetic hand ", MSc thesis submitted University of KwaZulu-Natal.

[21] Y.Shi. (Fabruary 2016)., "KNN Predictability Analysis Of Stock And Share Closing Prices",. University Of Leicester.

[22] B. A. M.King, (2015)., "Optimization Of Prosthetic Hand Manufacturing",. Optimization Of Prosthetic Hand Manufacturing (pp. 59-61). USA: Proceeding in IEEE Global Humanitarian Technology Conference.

[23] S. M. Roberts(2002), 'Investigation into the control of an upper-limb myoelectric prosthesis", university of plymouth.

[24] R. M. Howard(2002), "Investigation of Pattern Recognition System based On EMG Signals for optimal electrodes number and Positions" Boletin epidemiologico semanal, vol.10, no. 12, pp. 121124. 
[25] G. Beng. Lau(2009), "An Intelligent Prosthetic Hand Using Hybrid Actuation and myoelectric Control ", Phd thesis submitted to University of Leeds.

[26] A. A. Ibrahem(2019)," development of wearable intelligent mind controlled upper limp prosthetic by activates of brain" ,MSc thesis submitted to al-
Nahrain University, mechanical engineering department ,Iraq.

[27] Mohammed Z. Al-Faiz et al," A k-Nearest Neighbor Based Algorithm for Human Arm Movements Recognition Using EMG Signals" Iraq J. Electrical and Electronic Engineering Vol.6 No.2, 2010. 